\title{
Use of the World Wide Web in astronomy teaching
}

\author{
By Jay M. Pasachoff \\ Williams College-Hopkins Observatory Williamstown, Massachusetts 01267, USA
}

I discuss the burgeoning World Wide Web and how it can be used to aid astronomy teaching. I supply a list of a variety of useful Web sites.

The World Wide Web was invented 5 years ago at CERN, which is now translated as the European Laboratory for Particle Physics, as a way of aiding access to information from remote sites. The invention of graphic interfaces, notably Mosaic by a group at the National Supercomputer Center in Illinois and then Netscape Navigator as a private development by many of the original Mosaic people, led to an explosion in use of the Web. Millions of people around the world are now able to access information from over 100,000 Web sites.

There is much astronomical information on the Web, though that information make up only a small fraction of all the information available through this medium. The astronomical information is of many varied types, from images of observations to tables of data to lesson plans to journal articles. The question for us to address here is how best to make use of this information for astronomy teaching. Even with the increased resources available at our desktops, the individual teacher remains an important part of the educational enterprise.

One set of alternatives deals with whom the Web information is aimed at. To present new Web data in class, it is useful to have a means of projecting computer information on a screen, which is most often done with an LCD projector panel. An alternative is to use a color printer to make a transparency, a process that can now be done with a relatively inexpensive and portable setup. Another possible alternative is for the students to have their own direct access to the Web, directing their attention to sites to access from their own rooms or from some central locations.

The Web is most useful when you have access from a high-throughput source, such as the type of access known as a T1 line. Universities will often have such lines, which allow a Web page to appear on your screen in seconds. An intermediate quality line known as ISDN is sometimes available in the US, but is not in much use in the education market. Other people have to use ordinary modems, and even at the current maximum speed of $28,800 \mathrm{bps}$ it can take minutes to download individual Web pages, which makes the job of "surfing the Web"tedious. I think that only when everyone has access to the Web through T1 lines or equivalent will the system be truly widely useful. In the next years, access over cable TV lines may provide such universal high-speed access.

Of course, not all countries have good Internet access as of now. But as such access spreads, the Web will be a mechanism to bring in much information useful for astronomy teaching.

Individual Web sites begin with "homepages," the top level page of information that shows when someone signs onto your site. These homepages contain both text and graphics; new versions of Netscape Navigator allow motion of features on the pages, which may become unsuitably jazzy as a result. Still newer capabilities, such as those provided by Sun Microsystem's Java, allow control information to be sent out with the standard Web information, allowing still further customization. Web sites allow not only still images but also movies and sound to be downloaded.

A typical Web address, accessible through "Netscape,"might be:

http://www.yoursite.youruniversity.edu/teaching.html.

The address can be parsed as follows, with the "dots" as separators and read as "dot":

- "http://" stands for "hypertext transfer protocol,"the format by which data are transferred; all Web addresses begin with it, though it is not necessary always to type it.

- www is a common beginning for Web addresses.

- yoursite is the "server" computer that is holding the Web information.

- youruniversity is the computer your University uses for access to the Internet 
- "edu" is the suffix for U.S. educational sites; other U.S. suffixes are "gov" for government, "org"for organizations (like the American Astronomical Society), and "com"for commercial; sites in other countries end with two letter codes for those countries, such as "fr"for France.

- "/teaching" means that on the computer is a set of files known as "teaching"; you can have many documents within those files.

- ".html"stands for "hypertext markup language,"the computer language used.

I maintain a site at

\section{http://www.astro.williams.edu/jay}

at which, in coordination with my textbook Astronomy: From the Earth to the Universe, I maintain updates to a variety of astronomical topics as well as links to other Web sites around the world. Merely clicking on such "hotlinks" sends the computer to the other site. Netscape includes "forward" and "back"buttons to click to allow you quickly to see sites you have looked at recently. You can also enter a list of "bookmarks"that list sites by name rather than by the address; such bookmark lists quickly grow too long for easy use and then can be categorized by subtopics.

Transoceanic downloading of Web pages can take a lot of time, and increasing Web use has slowed transfer times worldwide. Some sites have "mirror sites" set up that will be closer to many users. The popular "The Nine Planets"site, for example, has a dozen mirror sites around the globe at which all the information is downloaded periodically.

A problem for Web use is that sites are sometimes "down" or else inaccessible because of delays or other problems on Internet lines. Thus it is not recommended that you require the Web for a class lecture. But you can download the information before class and store it on the hard disk of your computer for later replay.

Delays in the gratification of receiving Web information also mean that one cannot play the type of video games that are so popular. These delays leave an important niche for CD-ROMs (to be updated in capability in late 1996 by DVDs: digital video disks, with much more capacity). For example, an astronomy CD-ROM like Maris Multimedia's RedShift provides the capability of calculating the appearance of the sky from the Earth's surface or from positions elsewhere in the solar system (even close to another planet) for any time or date, as well as many hundreds of still images and a dozen short movies. I have worked with Maris on a further CD-ROM entitled Solar System Explorer, with still more capability for information about and images of our solar system. If the role of the Web is limited to providing a limited amount of supplemental information to such CD- ROMs, then there will be many fewer delays than by using the Web exclusively.

Though the works of Shakespeare and other authors out of copyright appear on the Web, contemporary works are less likely to be there because of the need of authors and publishers to be remunerated for their efforts. Thus for the moment, textbooks are not appearing on the Web, though supplemental information is. The whole Web is in a state of flux, and it is not yet possible to know how it will evolve. Some publishers like Time, Inc., with its magazines, and newspapers like The New York Times or The Times (London) are now available free on the Web, though they are likely to try to attract paying subscribers and cancel free access. Advertising appears on many homepages, but has not (yet?) proved to provide enough income to provide the large expenses of establishing and maintaining elaborate sites. Some attempts are under way to allow billing of small increments of Web access, such as the Clickshare process developed by a company in my hometown of Williamstown. We scientists and educators are used to free access to information on the Internet, but whether that no-cost access will continue is not now known. In any case, the Web provides powerful access to information around the world. Table 1 lists a number of Websites of astronomical interest; they are also available through hotlinks on my own site. Many of the sites also have hotlinks to the same sites, so what results is truly-what else-a web. The Figures show samples of interesting homepages.

At Williams College, we are proud to be the home of an educational tradition: it was put 150 years ago that the ideal education is Mark Hopkins (then our President) on one end of a log and a student at the other end. In some cases, modern technology like the World Wide Web can help teachers maintain one-on-one education though with larger classes and with access to the 
world outside and the Universe beyond. It is up to us as classroom teachers and as developers of laboratories, lesson plans, and other educational materials to guide astronomy on the World Wide Web and to guide students in how best to take advantage of it.

Table 1. World Wide Web Sites for Astronomy

A.A.O. IMAGES http://www.aao.gov.au/images.html

AAVSO http://www.aavso.org/index.html

ADS Astronomy and Astrophysics Abstract Service http://adsabs.harvard. edu/abstract_service.html

American Astronomical Society (and access to Astrophysical Journal) http://www.aas.org/

The American Physical Society http://aps.org/

The Astronomical Society of the Pacific http://www.physics.sfsu.edu/asp/asp.html AstroWeb: list of astronomy departments http://cdsweb.u-strasbg.fr/astroweb/dept.html Astronomical World Wide Web Resources http://stsci.edu/astroweb/net-www.html

Big Bear Solar Observatory (daily solar images) http://sundog.caltech.edu/

Chaisson Proto http://tthep2.phys.ttu.edu/dka100/alansill/chaisson/index.html

The Compton Observatory Science Support Center http://antwrp.gsfc.nasa.gov/

CTI Centre for Geography Home Page http://www.geog.le.ac.uk/cti/index.html

European Space Agency http://www.esrin.esa.it/

FUSE Home Page (future spacecraft for the uv) http://fuse.pha.jhu.edu/

Galileo countdown at Jupiter http://www.jpl.nasa.gov/galileo/countdown/

Online from Jupiter - Galileo quest http://quest.arc.nasa.gov/jupiter.html

Gemini 8-m Telescopes Project http://www.gemini.edu/

General Astronomy Information http://www.ast.cam.ac.uk/RGO/leaflets/

Global Oscillation Network Group - GONG http://helios.tuc.noao.edu/gonghome.html

History of Astronomy: General, Historians, Archaeoastronomy, Links http://aibn55.astro.unibonn.de:8000/ pbrosche/astoria.html

History of Astronomy: Mesopotamian astronomy http://ccwf.cc.utexas.edu/ hope/aneastro.html

Hubble Space Telescope (see also STScI, below) http://www.stsci.edu

Hyakutake (C/1996 B2) Sky \& Tel http://www.skypub.com/comets/hyaku3.html\# top

IAU: Central Bureau for Astronomical Telegrams http://cfa-www.harvard.edu/cfa/ps/cbat.html

IAU (International Astronomical Union) http://www.lsw.uni-heidelberg.de/iau.html

ISO (ESA's Infrared Space Observatory) http://isowww.estec.esa.nl/

Jupiter/Comet Collision FAQ [Frequently Asked Questions] - Post-Impact http://www.isc.tamu.edu/ astro/sl9/cometfaq2.html

Kronk - Comets and Meteor Showers http://medicine.wustl.edu/ kronkg/index.html

Links from Scott http://www.keele.ac.uk/depts/po/scott/003.htm

Links from Astronomy Magazine http://www.kalmbach.com/astro/HotLinks/HotLinks.html MIT X-Ray Timing Explorer Project http://space.mit.edu/XTE/XTE.html 
NASA K-12: Live from the Hubble Space Telescope

http://quest.arc.nasa.gov/livefrom /hst.html

NASA Jet Propulsion Laboratory (including Galileo images) http://www.jpl.nasa.gov/

The Nine Planets [many mirror sites exist]

http://seds.lpl.arizona.edu/nineplanets /nineplanets/nineplanets.html

NSSDC home page [lots of data and images] http://nssdc.gsfc.nasa.gov/

Pasachoff's Astronomy Text Updates and Links http://www.astro.williams.edu/jay

Royal Astronomical Society http://www.star.ucl.ac.uk/ jl/mypage.html

SDAC - NASA Solar Home Page http://umbra.gsfc.nasa.gov/sdac.html

SkyView home page http://skyview.gsfc.nasa.gov/skyview.html

SoHO - The Solar and Heliospheric Observatory http://sohowww.nascom.nasa.gov/

Solar Eclipse Images http://umbra.nascom.nasa.gov/eclipse/images/eclipse_images.html

Solar Image Index http://www.sel.noaa.gov/images/

SolarNews - Index http://helios.tuc.noao.edu/SolarNews/index.html

Space Physics Homepage http://umbra.nascom.nasa.gov/spd/spd.html

STScI Press Releases http://www.stsci.edu/pubinfo/PR.html

STScI/HST Public Information http://www.stsci.edu/public.html

Stardust NASA Comet Mission http://pdcsrva.jpl.nasa.gov/stardust/home.html

StarWorlds - Astronomy and Related Organizations http://cdsweb.u-strasbg.fr/ heck/sfworlds.htm

SXT Home page-Lockheed/Yohkoh (X-ray Solar Images)

http://pore1.space.lockheed.com:80/SXT/

Sun: Granulation movies http://www.erim.org/algs/PD/pd_home.html

Sunspot number SIDC http://www.oma.be/KSB-ORB/SIDC/index.html

Sunspot/butterfly graph: Marshall Space Flight Center/Solar Physics Branch http://wwwssl.msfc.nasa.gov/ssl/pad/solar/

Transneptunian Object List http://cfa-www.harvard.edu/cfa/ps/lists/TNOs.html

Ulysses/ESA http://www.esoc.esa.de/external/mso/ulysses.html

Ulysses/JPL http://ulysses.jpl.nasa.gov/

USGS's list of astronomy links http://info.er.usgs.gov/network/science/ astronomy/index.html

USGS/JPL Planetary [access to prints and slides of planetary images] http://acheron.jpl.nasa.gov/PIA/PIA/html

Yahoo - Science: Astronomy [a Web Crawler/search engine] http://beta.yahoo.com/Science/Astronomy/ 

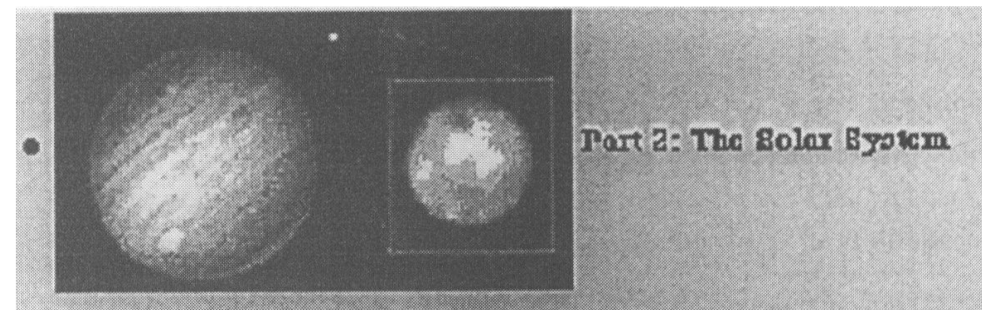

- Claptor 6: 'Tha structum ani urme at the solar syatem

o Cintor 7: 0nikerth

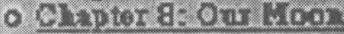

- Clepter ]= Hercerr.

- Clepter 10: 7oxm

o Clapter 11: Riars:

o Olupter 12: Jopieter

o Olapter 12: gatorn

u G)

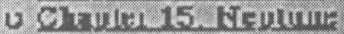

13 Glauini 16. Pluii:

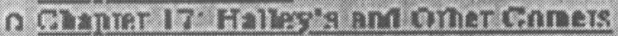

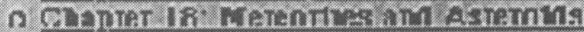

o Clapter 19: Late in the Uimerse

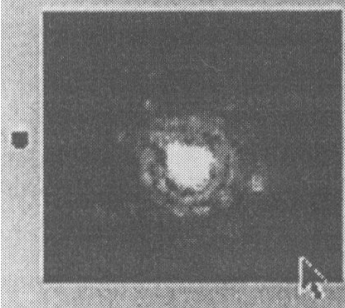

Twe Stan:

- Clapter 20: Oxdinary 8tary

o Clapter 21: Brcllar Dismnices and Mrations

- Olapter 22: Doubles. Yoriables and Clasters

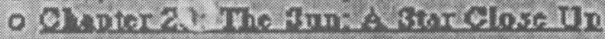

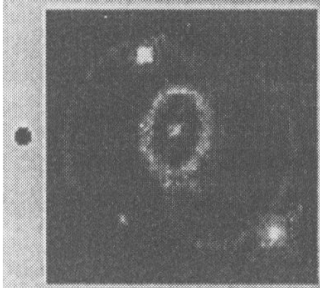

Hart 4: Stailar KFolnton

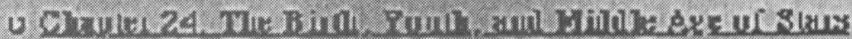

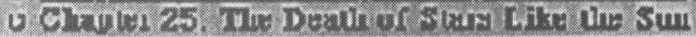

FIgure 1. Part of Pasachoff On-Line, http://www.astro.williams.edu/jay. Updates and hotlinks. As always, underlined text represents hotlinks; clicking on an underlined word takes you to the other site. 


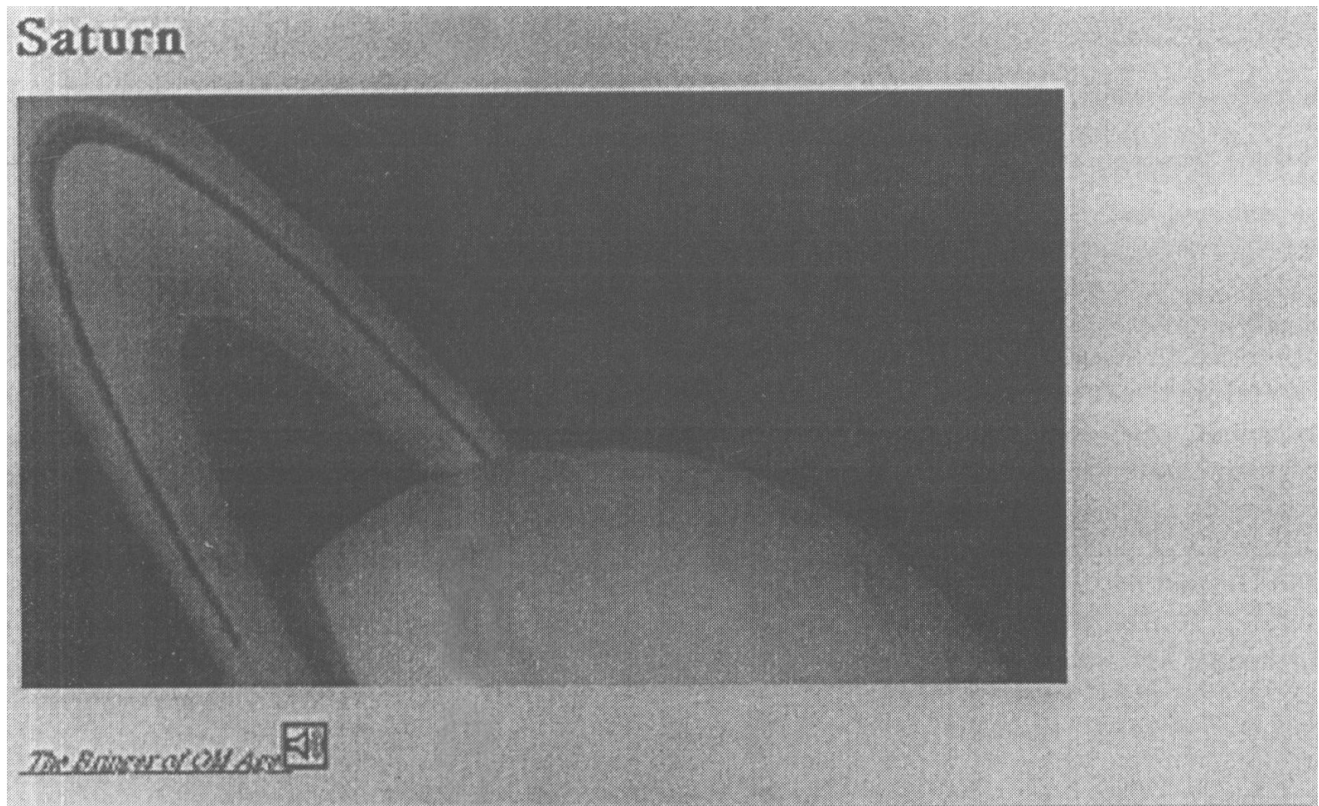

\section{Saturn Facts}

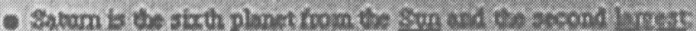
a distunce from sun $1,09,00,000 \mathrm{~km}(9.54 \mathrm{LU}$

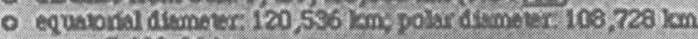
0 mass: $5.68006 \mathrm{kz}$

- In Roman mythobgy, Satum ts the god of erriculture. The associated Greek god, Cronts, was one son of

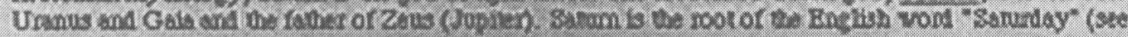
Abretits 4 .

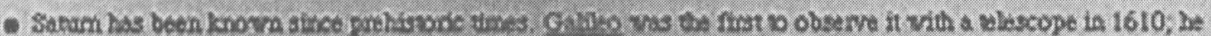

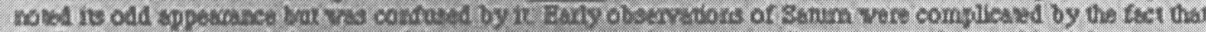

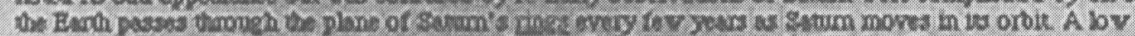

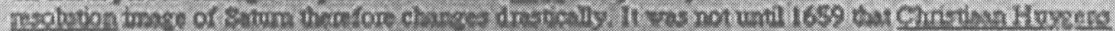

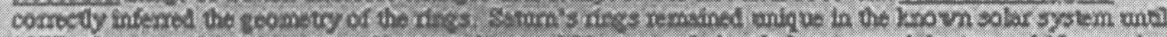

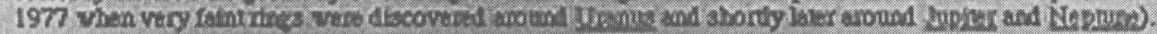

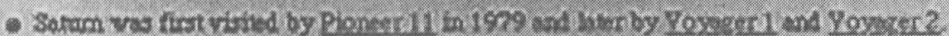

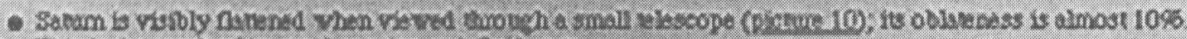

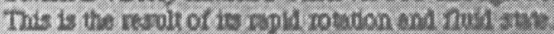

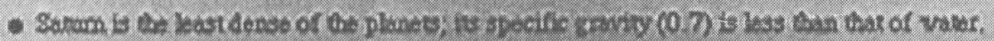

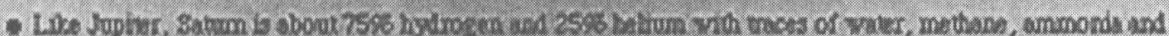

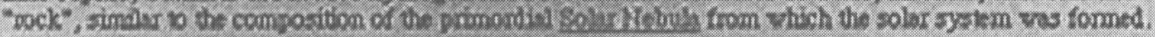

Figure 2. The Saturn page from "The Nine Planets": http://seds.lpl.arizona.edu/nineplanets/nineplanets/nineplanets.html. 